\title{
Growth-interruption-induced low-density InAs quantum dots on GaAs
}

\author{
L. H. Li, ${ }^{1, a)}$ N. Chauvin, ${ }^{2}$ G. Patriarche ${ }^{3}$ B. Alloing, ${ }^{1, b)}$ and A. Fiore ${ }^{2}$ \\ ${ }^{1}$ Ecole Polytechnique Fédérale de Lausanne, Institute of Photonics and Quantum Electronics, Station 3, \\ CH-1015 Lausanne, Switzerland \\ ${ }^{2}$ COBRA Research Institute, Eindhoven University of Technology, P.O. Box 513, 5600 MB Eindhoven, \\ The Netherlands \\ ${ }^{3}$ LPN/CNRS, Route de Nozay, 91460 Marcoussis, France
}

(Received 13 July 2008; accepted 28 August 2008; published online 21 October 2008)

\begin{abstract}
We investigate the use of growth interruption to obtain low-density InAs quantum dots (QDs) on GaAs. The process was realized by Ostwald-type ripening of a thin InAs layer. It was found that the optical properties of the QDs as a function of growth interruption strongly depend on InAs growth rate. By using this approach, a low density of QDs $\left(4 \mathrm{dots} / \mu \mathrm{m}^{2}\right)$ with uniform size distribution was achieved. As compared to QDs grown without growth interruption, a larger energy separation between the QD confined levels was observed, suggesting a situation closer to the ideal zero-dimensional system. Combining with an InGaAs capping layer such as In-rich QDs enable $1.3 \mu \mathrm{m}$ emission at 4 K. () 2008 American Institute of Physics. [DOI: 10.1063/1.3000483]
\end{abstract}

The growth optimization of self-assembled quantum dots (QDs) on GaAs has been focused first on the realization of low-threshold lasers. More recently, it has been demonstrated that QDs can be efficiently used as single-photon emitters for quantum cryptography applications ${ }^{1,2}$ and growth efforts have been devoted to the fabrication of low-density QD samples to perform single dot spectroscopy. Fiber-based quantum communication applications require an emission wavelength in the 1.3 or $1.55 \mu \mathrm{m}$ transmission window. However, most studies have concentrated on QDs emitting in the $\lambda<1 \mu \mathrm{m}$ range. This is due to the lower sensitivity of detectors and also to the difficulties in growing sparse and large enough QDs emitting in this spectral region. There are few reports of microphotoluminescence (micro-PL) and antibunching experiments based on $1.3 \mu \mathrm{m}$ InAs/GaAs QDs. ${ }^{3,4}$ In particular, we demonstrated that a uniform distribution of low-density InAs QDs emitting at $1.3 \mu \mathrm{m}$ with high efficiency can be obtained by employing a combination of ultralow InAs growth rate and InGaAs capping layer., Clean exciton-biexciton dynamics, ${ }^{5}$ antibunching, ${ }^{4}$ and electroluminescence from single QDs (Ref. 7) were observed, making these QDs promising candidates for single-photon sources at $1.3 \mu \mathrm{m}$. However, due to the different growth rates of InAs QDs and InGaAs capping layer, two In cells have to be used for the growth of such long-wavelength QDs, ${ }^{5,6}$ which reduces the flexibility of the QD growth. Lowdensity InAs QDs can also be obtained by growing a thin layer of InAs close to the critical thickness of the two- (2D) to three-dimensional (3D) growth mode transition. ${ }^{8,9}$ However, in this case it is difficult to reach $1.3 \mu \mathrm{m}$ wavelength emission since this requires large and thick QDs, i.e., a relatively large amount of InAs. The Ostwald-type ripening process occurring during a growth interruption enables the

\footnotetext{
${ }^{\text {a) }}$ Present address: School of Electronic and Electrical Engineering, The University of Leeds, Woodhouse Lane, Leeds LS2 9JT, UK. Electronic mail: 1.h.li@leeds.ac.uk.

${ }^{b)}$ Present address: Laboratorium für Festkörperphysik ETH Zürich, CH-8093 Zürich, Switzerland.
}

growth of larger QDs on the expense of smaller QDs and potentially also the wetting layer (WL) underneath the QDs, ${ }^{10-15}$ which creates an opportunity to extend the emission wavelength of such QDs. Indeed, significant PL energy redshift from the QDs formed by Ostwald-type ripening of a 1.8 monolayer (ML) thickness of InAs layer has been observed. ${ }^{12,13}$ Furthermore, reduction in the QD density was also demonstrated. ${ }^{10,15}$ In this work, we show that by combining a low growth rate and a growth interruption it is possible to obtain low-density and In-rich QDs with very uniform size distribution. The QDs obtained by this technique show higher confinement energies and larger separation between the energy levels as compared to our previous method. ${ }^{5}$ Additionally, as the indium cell temperature can be changed during the growth interruption, the QDs and the InGaAs capping layer can be grown with the same cell, releasing the need for two cells. Emission from single QDs in the $1.3 \mu \mathrm{m}$ wavelength range at $4 \mathrm{~K}$ is demonstrated using this combination of low growth rate, ripening, and capping.

The samples were grown by molecular-beam epitaxy (MBE) on semi-insulating (001) GaAs substrates. After oxide desorption, a $0.5-\mu \mathrm{m}$-thick GaAs buffer layer was grown at $620{ }^{\circ} \mathrm{C}$. Then, a $1.7 \mathrm{ML}$ InAs layer was deposited at $500{ }^{\circ} \mathrm{C}$. After growth of the InAs layer, a growth interruption varying from 0 to $240 \mathrm{~s}$ was applied. During the growth interruption, the growth temperature was kept the same as that of the QD growth to allow Ostwald-type ripening. ${ }^{10-15}$ The InAs thickness was determined by the observation of 2D-3D transition. ${ }^{8-10}$ Once the onset of the 2D-3D transition was observed, the nominal InAs critical thickness of about 1.7 ML was achieved. The samples were completed by a 100-nm-thick GaAs cap. Their structural and optical properties were characterized by atomic force microscopy (AFM), transmission electron microscopy (TEM), and PL measurements.

In order to demonstrate the evolution of the optical properties of the samples as a function of growth interruption, two sets of samples were grown at different InAs growth 


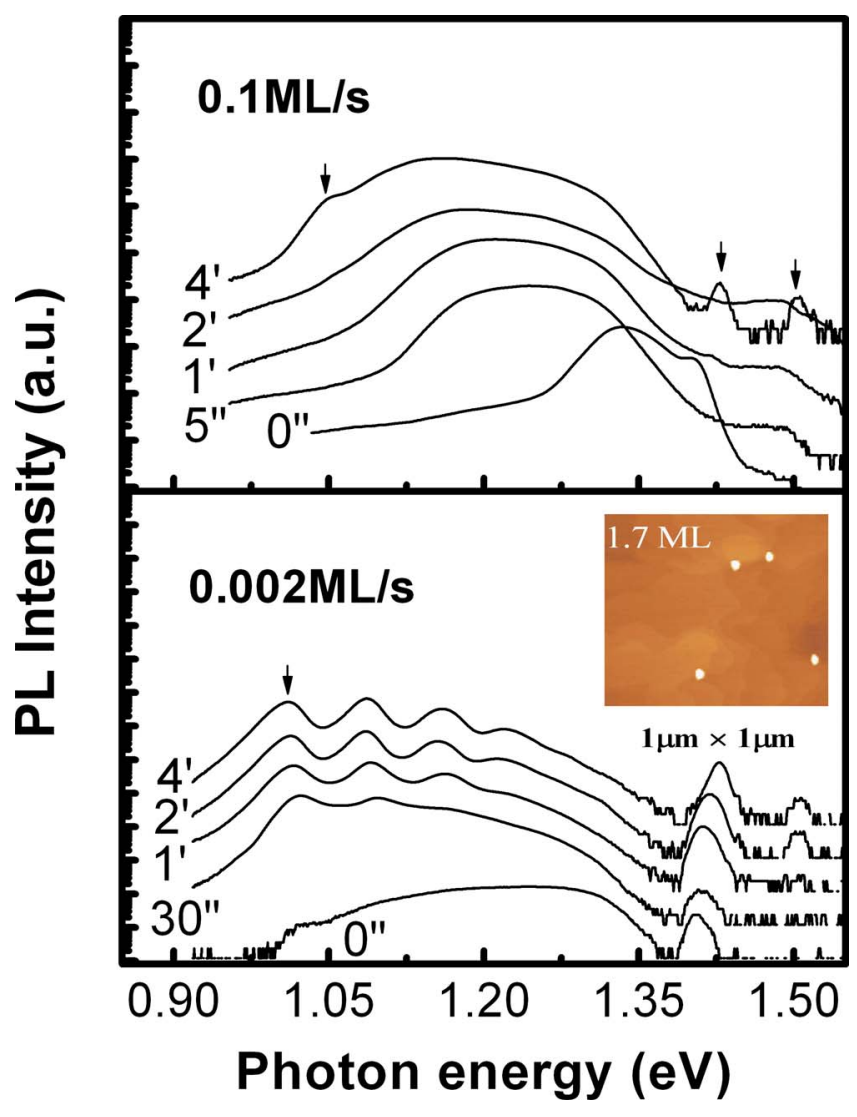

FIG. 1. (Color online) $77 \mathrm{~K} \mathrm{PL}$ spectra of the samples grown at different InAs growth rates of 0.002 and $0.1 \mathrm{ML} / \mathrm{s}$, respectively. After the growth of the InAs layer, different growth interruptions were used. The curves have been shifted vertically for clarity. Inset: AFM of a reference sample grown with a growth rate of $0.002 \mathrm{ML} / \mathrm{s}$ and growth interruption of $120 \mathrm{~s}$.

rates of 0.002 and $0.1 \mathrm{ML} / \mathrm{s}$, respectively. Figure 1 shows the 77 K PL spectra of these samples. The PL spectra of samples grown at higher growth rate are dominated by a broad PL band, as shown in Fig. 1(a). The observation is very similar to the reports in Refs. 12 and 13. In contrast, for the samples grown at lower growth rate, clear PL peaks associated with InAs QDs are present in all spectra except for the one without growth interruption as shown in Fig. 1(b). Without growth interruption, a broad PL band covering 1.0-1.37 eV was observed, similar to that of the sample grown at higher growth rate. We are working around the regime of 2D-3D transition. As a consequence, a high density of precursors coexists with QDs of different sizes for the samples grown without growth interruption. ${ }^{16}$ The size dispersion results in the broad PL bands observed in these samples. Growth interruption improves the QD size distribution, but the improvement is completely different for the samples grown at different growth rates, probably due to the strong growth rate dependence of the QD size and density. ${ }^{14,17}$ At lower growth rate, the QD mean size becomes larger while the QD density and size fluctuation are reduced significantly. ${ }^{14}$ The increase in size and decrease in size fluctuation favor the reduction in the inhomogeneous linewidth of the emission. Therefore, it is essential to use a low growth rate to obtain a narrow QD size distribution. QDs in an uncapped reference sample grown with a growth rate of $0.002 \mathrm{ML} / \mathrm{s}$ and growth interruption of $120 \mathrm{~s}$ have a density of about $4 \mathrm{dots} / \mu \mathrm{m}^{2}$. AFM shows that

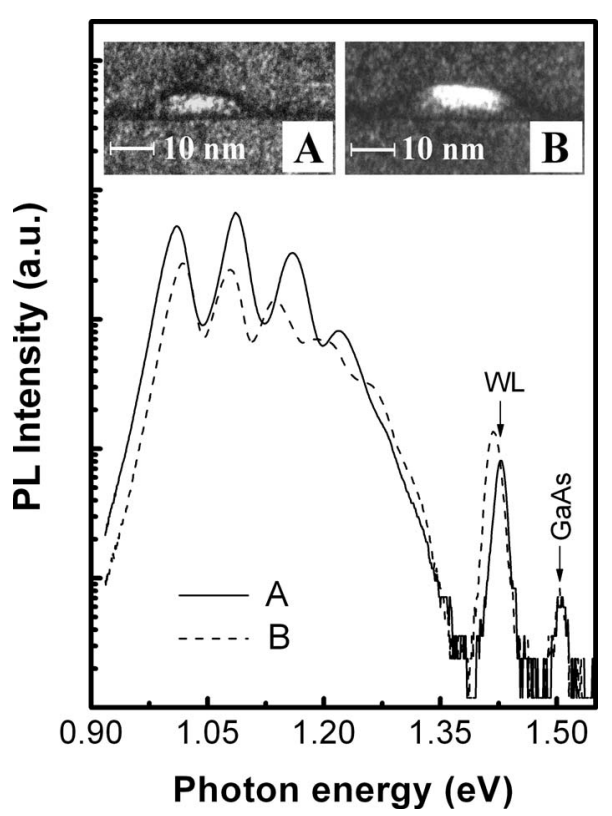

FIG. 2. $77 \mathrm{~K}$ PL spectra of two samples (A and B) grown at the same InAs growth rate of $0.002 \mathrm{ML} / \mathrm{s}$. The differences between these samples are InAs thicknesses and growth interruption. Sample A: 1.7 ML InAs layer with a growth interruption of $240 \mathrm{~s}$, sample B: $2.1 \mathrm{ML}$ InAs layer without growth interruption. Insets show the $g=\langle 002\rangle$ dark field cross-sectional TEM images of these samples.

the QDs are very uniform and have a single-mode size distribution (see inset). With increasing growth interruption, the WL peak blueshifts and the QD peaks/band redshift. Such anticorrelation behavior has been explained by mass transfer from the 2D layer to 3D islands. ${ }^{11,18}$ The combined and opposite shifts in the QD and WL peaks suggest that the QD formation significantly consumes the WL. This is consistent with the report in Refs. 10 and 14 where significant QD volume density increase was observed. With increasing growth interruption, the PL peaks become well developed and separated, particularly for the samples grown at low growth rate. This phenomenon is attributed to the increased QD size uniformity due to Ostwald-type ripening. ${ }^{10-15}$

The ground state emission energy of the QDs grown by ripening of low-growth-rate InAs thin layer is about $1.0 \mathrm{eV}$ at $77 \mathrm{~K}$. The value corresponds to a room temperature emission of about $1.3 \mu \mathrm{m}$ (spectrum not shown). Usually, room temperature emission up to $1.3 \mu \mathrm{m}$ from the InAs QDs embedded in GaAs matrix can only be achieved by growing thick InAs layer ( $>2 \mathrm{ML}$ ) with a very low growth rate, provided that growth interruption and InGaAs capping layer are not used. ${ }^{4,5}$ This unusual increase in the emission wavelength makes it interesting to compare the QDs grown with different approaches. Figure 2 shows the $77 \mathrm{~K}$ PL spectra of two samples (A and B) grown at the same growth rate of $0.002 \mathrm{ML} / \mathrm{s}$. The differences between these samples are InAs thicknesses and growth interruption. Sample A including 1.7 ML InAs was grown with a growth interruption of $240 \mathrm{~s}$ while sample B including 2.1 ML InAs was grown without growth interruption. Interestingly, the InAs thicknesses are different, but their ground state energies are nearly the same (around $1.018 \mathrm{eV}$ ). Compared to sample B, the WL peak of sample $\mathrm{A}$ is $18 \mathrm{meV}$ higher in energy, indicating that the 


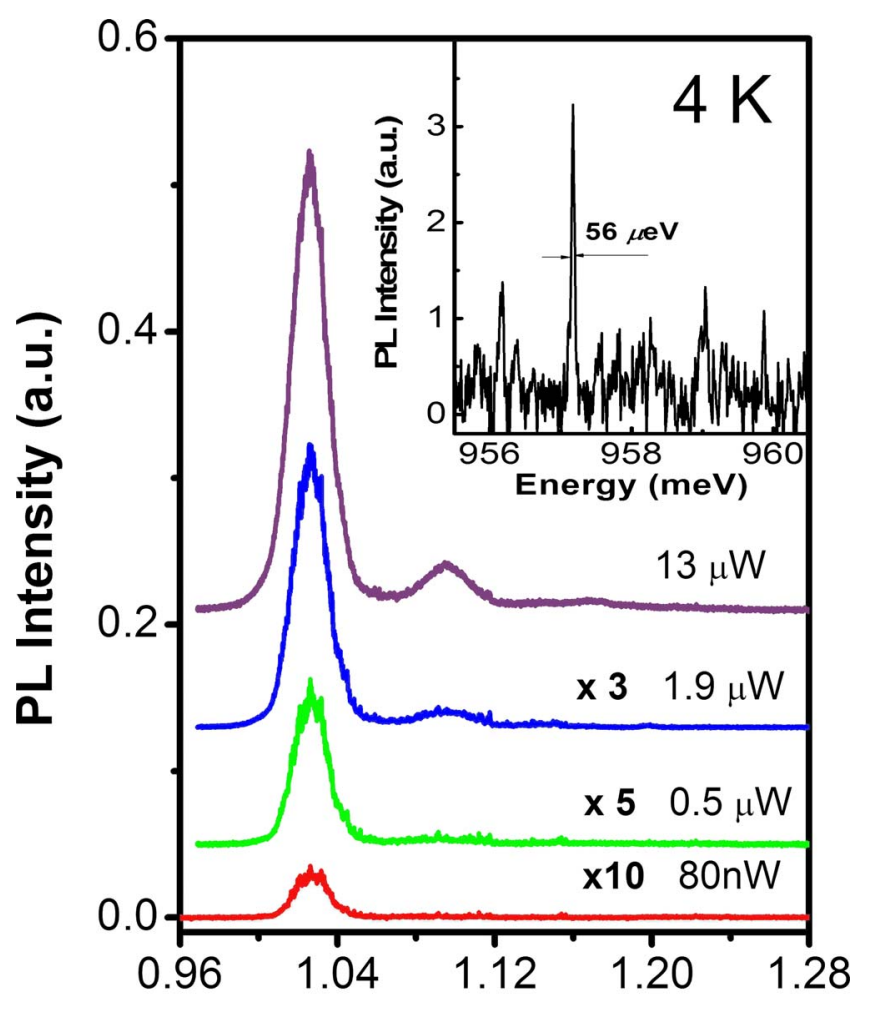

Photon Energy (eV)

FIG. 3. (Color online) Typical $4 \mathrm{~K}$ PL spectra of the sample grown with an InAs growth rate of $0.002 \mathrm{ML} / \mathrm{s}$ and a growth interruption of $120 \mathrm{~s}$. The spectra were taken at different excitation powers. Inset: $4 \mathrm{~K}$ micro-PL spectrum from an unpatterned InGaAs-capped sample.

ripening process used for sample A results in a WL, which is thinner or has a lower In content. The spectral separations between ground state and first excited state of the two samples are rather different (73 and $61 \mathrm{meV}$ for samples A and $\mathrm{B}$, respectively). The nearly equal ground state energies and appearance of a larger energy difference can be attributed to simultaneous size decrease and In content increase in the QDs in sample A. The QD size decrease was verified by TEM measurements as shown in the insets, where the $g$ $=\langle 002\rangle$ dark field cross-sectional TEM images of the samples are presented. The QD dimensions (base length $\times$ height) in sample A are about $22 \mathrm{~nm} \times 5 \mathrm{~nm}$ while the QD dimensions in sample $B$ are about $35 \mathrm{~nm} \times 7 \mathrm{~nm}$. The In content increase in the QDs can be understood on the basis of enhanced In adatom migration. ${ }^{10,14}$ Indeed, during the growth interruption, In adatoms have enough time to migrate on the growth surface, which favors the incorporation into existing coherent islands. As a consequence, the In content of the QDs increases and the same ground state energy is obtained with smaller QDs. The QDs with larger energy spacing between ground state and excited states, including the WL, represent a situation closer to the ideal zero-dimensional system and we expect improved characteristics (e.g., increased temperature stability) in single-photon devices incorporating such QDs.

Typical $4 \mathrm{~K}$ PL spectra of the sample grown with InAs growth rate of $0.002 \mathrm{ML} / \mathrm{s}$ and growth interruption of $120 \mathrm{~s}$ are presented in Fig. 3. The spectra were taken at different excitation powers. For the spectra taken at lower excitation powers, only single Gaussian peak with a linewidth of 17 $\mathrm{meV}$ is observed, indicating that the size of the QDs is very uniform. ${ }^{19}$ The observation is consistent with AFM investigations. With increasing excitation power, a high energy peak arises due to state filling behavior of the QDs. ${ }^{20}$ The emission wavelength of the QDs is about $1.2 \mu \mathrm{m}(1.038 \mathrm{eV})$ at $4 \mathrm{~K}$, but it can be easily extended up to $1.3 \mu \mathrm{m}$ by using an InGaAs capping layer, as discussed in Refs. 5 and 6. The inset shows the $4 \mathrm{~K}$ micro-PL emission spectrum from an unpatterned InGaAs-capped sample, which was grown with a single In cell for both the QDs and the capping layer. Sharp isolated lines with a linewidth of about $56 \mu \mathrm{eV}$ at 957.2 $\mathrm{meV}$ are observed, not only confirming the low-density and high optical quality of these QDs, but also suggesting their potential application to $1.3 \mu \mathrm{m}$ single-photon sources.

In conclusion, MBE growth of InAs QDs by Ostwaldtype ripening of thin InAs layer was investigated. The optical properties of the QDs as a function of growth interruption are shown to strongly depend on InAs growth rate. Low-density QDs with a narrow size distribution can be obtained by the ripening of an InAs layer with the critical thickness value, if this is grown at low growth rate. As a consequence of the ripening process, QDs with larger confinement energy and energy spacing between bound states are formed, representing a situation closer to the ideal zero-dimensional system. Combining with an InGaAs capping layer, ripening of a lowgrowth-rate thin InAs layer enables $4 \mathrm{~K}$ emission from single QDs with wavelength around $1.3 \mu \mathrm{m}$, which provide an option in realizing single-photon emitters for potential fiberbased quantum communication applications.

We acknowledge financial support from the Swiss Commission for Technology and Innovation (CTI-TOPNANO21 program), the OFES (COST program), and the Swiss National Science Foundation.

${ }^{1}$ P. Michler, A. Kiraz, C. Becher, W. V. Schoenfeld, P. M. Petroff, L. Zhang, E. Hu, and A. Imamoglu, Science 290, 2282 (2000).

${ }^{2}$ C. Santori, M. Pelton, G. Salomon, Y. Dale, and Y. Yamamoto, Phys. Rev. Lett. 86, 1502 (2001).

${ }^{3}$ M. B. Ward, O. Z. Karimov, D. Unitt, Z. L. Yuan, P. See, D. G. Gevaux, A. J. Shields, P. Atkinson, and D. Ritchie, Appl. Phys. Lett. 86, 201111 (2005).

${ }^{4}$ C. Zinoni, B. Alloing, C. Monat, V. Zwiller, L. H. Li, A. Fiore, L. Lunghi, A. Gerardino, H. de Riedmatten, H. Zbinden, and N. Gisin, Appl. Phys. Lett. 88, 131102 (2006).

${ }^{5}$ B. Alloing, C. Zinoni, V. Zwiller, L. H. Li, C. Monat, M. Gobet, G. Buchs, A. Fiore, E. Pelucchi, and E. Kapon, Appl. Phys. Lett. 86, 101908 (2005).

${ }^{6}$ B. Alloing, C. Zinoni, L. H. Li, A. Fiore, and G. Patriarche, J. Appl. Phys. 101, 024918 (2007).

${ }^{7}$ C. Monat, B. Alloing, C. Zinoni, L. H. Li, and A. Fiore, Nano Lett. 6, 1464 (2006).

${ }^{8}$ D. Leonard, K. Pond, and P. M. Petroff, Phys. Rev. B 50, 11687 (1994).

${ }^{9}$ F. Patella, F. Arciprete, M. Fanfoni, A. Balzarotti, and E. Placidi, Appl. Phys. Lett. 88, 161903 (2006).

${ }^{10}$ T. J. Krzyzewski and T. S. Jones, J. Appl. Phys. 96, 668 (2004).

${ }^{11}$ L. Müller-Kirsch, R. Heitz, U. W. Pohl, D. Bimberg, I. Häusler, H. Kirmse, and W. Neumann, Appl. Phys. Lett. 79, 1027 (2001).

${ }^{12}$ U. W. Pohl, K. Pötschke, A. Schliwa, F. Guffarth, D. Bimberg, N. D. Zakharov, P. Werner, M. B. Lifshits, V. A. Shchukin, and D. E. Jesson, 
Phys. Rev. B 72, 245332 (2005).

${ }^{13}$ S. Kiravittaya, Y. Nakamura, and O. G. Schmidt, Physica E (Amsterdam) 13, 224 (2002).

${ }^{14}$ P. B. Joyce, T. J. Krzyzewski, G. R. Bell, T. S. Jones, S. Malik, D. Childs, and R. Murray, Phys. Rev. B 62, 10891 (2000).

${ }^{15}$ D. Z. Hu, D. M. Schaadt, and K. H. Ploog, J. Cryst. Growth 293, 546 (2006).

${ }^{16}$ T. J. Krzyzewski, P. B. Joyce, G. R. Bell, and T. S. Jones, Phys. Rev. B 66, 121307(R) (2002).
${ }^{17}$ Y. Nakata, K. Mukai, M. Sugawara, K. Ohtsubo, H. Ishikawa, and N. Yokoyama, J. Cryst. Growth 208, 93 (2000).

${ }^{18}$ F. Heinrichsdorff, A. Krost, M. Grundmann, D. Bimberg, F. Bertram, J. Christen, A. Kosogov, and P. Werner, J. Cryst. Growth 170, 568 (1997).

${ }^{19}$ R. P. Mirin, K. L. Silverman, D. H. Christensen, and A. Roshko, J. Vac. Sci. Technol. B 18, 1510 (2000).

${ }^{20}$ S. Raymond, S. Fafard, P. J. Poole, A. Wojs, P. Hawrylak, S. Charbonneau, D. Leonard, R. Leon, P. M. Petroff, and J. L. Merz, Phys. Rev. B 54, 11548 (1996). 